\title{
List of Participants - IAU Colloquium 170
}

\begin{tabular}{|c|c|c|c|}
\hline Name & Country & Name & Country \\
\hline Aikman, Chris & Canada & Al-Malki, Mohammed & Saudi Arabia \\
\hline Baranne, Andre & France & Barban, Caroline & France \\
\hline Batten, Alan & Canada & Bernstein, Hans-Heinrich & Germany \\
\hline Bouchy, Francois & France & Brown, Tim & U.S.A. \\
\hline Cameron, Andrew & U.K. & Cardinal, Rob & Canada \\
\hline Cochran, Anita & U.S.A. & Cochran, William & U.S.A. \\
\hline Contos, Adam & U.S.A. & Cummings, Irene & New Zealand \\
\hline Davis, John & Australia & Desidera, Silvano & Italy \\
\hline Dravins, Dainis & Sweden & Ecker, George & Israel \\
\hline Fekel, Frank & U.S.A. & Fischer, Debra & U.S.A. \\
\hline Fletcher, Murray & Canada & Gray, David & Canada \\
\hline Griffin, Elizabeth & U.K. & Gullberg, Dag & Sweden \\
\hline Hajian, Arsen & U.S.A. & Hamilton, Devon & Canada \\
\hline Hatzes, Artie & U.S.A. & Hearnshaw, John & New Zealand \\
\hline Horner, Scott & U.S.A. & Hummel, Christian & U.S.A. \\
\hline Ilyin, Ilya & Finland & Irwin, Alan & Canada \\
\hline Isaak, George & U.K. & Kaszás, Gabor & Hungary \\
\hline Khalesseh, Bahram & Iran & Kürster, Martin & Chile \\
\hline Lampens, Patricia & Belgium & Larson, Ana & U.S.A. \\
\hline Latham, David & U.S.A. & Lester, John & Canada \\
\hline Marcy, Geoff & U.S.A. & Mayor, Michel & Switzerland \\
\hline Mazeh, Tsevi & Israel & McCarthy, Chris & U.S.A. \\
\hline McMillan, Robert & U.S.A. & Merline, William & U.S.A. \\
\hline Mkrtichian, David & Ukraine & Nisenson, Peter & U.S.A. \\
\hline Nordgren, Tyler & U.S.A. & Noyes, Robert & U.S.A. \\
\hline Popper, Dan & U.S.A. & Pourbaix, Dimitri & Belgium \\
\hline Queloz, Didier & U.S.A. & Ramsey, Larry & U.S.A. \\
\hline Richardson, Derek & U.S.A. & Robb, Russell & Canada \\
\hline Rucinski, Slavek & Canada & Scarfe, Colin & Canada \\
\hline Skuljan, Jovan & New Zealand & Soderblom, David & U.S.A. \\
\hline Stefanik, Robert & U.S.A. & Stickland, David & U.K. \\
\hline Szabados, Laszlo & Hungary & Szécsényi-Nagy, Gabor & Hungary \\
\hline Tokovinin, Andrei & Russia & Udry, Stephane & Switzerland \\
\hline Verschueren, Werner & Belgium & Vinkó, Joszef & Hungary \\
\hline Walker, Gordon & Canada & Yang, Stephenson & Canada \\
\hline
\end{tabular}

